\title{
Cinética de la inmunoglobulina-A salival (IgAs) en adultos jóvenes con capacidad aeróbica promedio o excelente antes y después de una prueba de esfuerzo máximo
}

\author{
Paulina Yesica Ochoa-Martínez, Javier Arturo Hall-López, Marco Antonio Martínez-García, \\ Raúl Díaz-Molina, Ana María Miranda Botelho Teixeira y José Antonio Moncada-Jiménez
}

Salivary immunoglobulin A (sIgA) kinetics in young adults with an average or excellent aerobic capacity before and after a cardiopulmonary graded exercise test

Background: Aim: To compare the concentration of secretory immunoglobulin-A (sIgA) in young adults with average or excellent aerobic capacity before and after a cardiopulmonary graded exercise test. Methods: Participants were nine apparently healthy physically active males (Mean age $=21.3 \pm 2.1 \mathrm{yr}$.), randomly allocated in two groups based on their $\mathrm{VO}_{2}$ max: a) average aerobic capacity $(\mathrm{AEC}, \mathrm{n}=5)$ or b) excellent aerobic capacity (EAC, $\mathrm{n}=4$ ). Participants performed the Bruce protocol to determine their aerobic capacity. The $\operatorname{sgA}$ was measured before the test, immediately after the test and 60-, 120-, 240-, and 1440-min after the test. Results: Mixed factorial $2 \times 6$ ANOVA indicated no significant interactions between groups and measurements $(\mathrm{p}=0.956)$, and main effect groups on $\operatorname{sIgA}(\mathrm{AEC}=85.4 \pm 19.3 \mu \mathrm{g} / \mathrm{mL}$ vs. $\mathrm{EAC}=79.2 \pm 21.5 \mu \mathrm{g} / \mathrm{mL}, \mathrm{p}=0.836)$. Tukey's post hoc analysis revealed significant differences measurement obtained immediately after the test and between the initial measurement $(p=0.020), 60-\min (p=0.030), 240-\min (p=0.016)$, and 1440-min $(p=0.028)$ following the test. Conclusion: There is no change in SIgA kinetics depending on the aerobic capacity of the participants following an aerobic capacity cardiopulmonary graded exercise test.

Key words: Secretory immunoglobulin-A, maximal oxygen uptake, cardiopulmonary exercise test.

Palabras clave: Inmunoglobulina A salival, consumo máximo de oxígeno, prueba de esfuerzo.

\section{Introducción}

$\mathrm{D}$ e acuerdo al Colegio Americano de Medicina del Deporte (ACSM) y el Colegio Europeo de Ciencias del Deporte (ECSS), la valoración inmunológica ha sido comúnmente utilizada como parámetro fisiológico para determinar el síndrome de sobreentrenamiento ${ }^{1}$, específicamente la inmunoglobulina A. Esta inmunoglobulina constituye una de las primeras barreras de defensa de los seres humanos en sangre ${ }^{2} \mathrm{y}$ en las mucosas actúa como la defensa inicial contra los patógenos invasores (virus y bacterias) antes de que penetren en el plasma, identificando a los antígenos patógenos e impidiendo que se instalen en las mucosas ${ }^{3}$. Los mecanismos que hacen funcionar al sistema inmunológico disminuyen con el sobre-entrenamiento, como la producción de linfocitos $\mathrm{T}$ y $\mathrm{B}^{2}$. En las ciencias aplicadas al deporte, la cantidad de investigaciones sobre temas inmunológicos se han incrementado en los últimos años; sus resultados han comprobado que los beneficios del ejercicio moderado mejoran las funciones del sistema inmunológico pero en el caso de atletas de alto rendimiento se ha reportado que presentan más probabilidad de riesgo de contagio de infecciones, principalmente del tracto respiratorio superior (TRS $)^{4}$. Investigaciones refieren mayor cantidad de episodios y síntomas de enfermedades infecciosas del TRS en atletas de alto rendimiento, en comparación con personas moderadamente activas ${ }^{5,6}$.

Estudios de tipo longitudinal y revisiones sistemáticas muestran claramente que altas demandas de preparación y cargas de entrenamiento de los deportistas de alto rendimiento tienen la finalidad de producir un desequilibrio controlado de la homeostasis para generar adaptaciones biológicas y a su vez mejorar el rendimiento físico ${ }^{7}$, pero esto pueden influenciar que ese estrés se acumule por cargas extenuantes de entrenamiento, afecte la función inmunológica de los atletas y por ende, su salud ${ }^{8}$ siendo esto una situación susceptible a infecciones oportunistas que para contrarrestarlas requerirían reposo, detener el programa de entrenamiento o no competir en un evento deportivo ${ }^{2,5,6}$; Por lo anterior las aplicaciones prácticas para los atletas, entrenadores y su personal de apoyo médico es buscar directrices para reducir el riesgo de la enfermedad $^{9,10}$.
Universidad Autónoma de Baja California. México.

Facultad de Deportes (PYO, JAH). Laboratorio de Biociencias de la Motricidad Humana-LABIMH (MAM).

Facultad de Medicina (RD) Universidad de Costa Rica. Costa Rica.

Escuela de Educación Física y Deporte y del Centro de Investigación en Ciencias del Movimiento Humano (CIMOHU) (AMMBT).

Universidad de Coimbra, Portugal.

Facultad de Ciencias del Deporte y Educación Física (JAM).

La presente investigación contó como fuente de apoyo financiero a la convocatoria del Programa de Intercambio y Cooperación Internacional de Estudios entre Europa y Sudamérica Erasmus Mundus PRECIOSA 2014 y 2015 y a la convocatoria proyectos de investigación por unidad académica de la Universidad Autónoma de Baja California UABC; Registro: 49/1405.

Los autores declaran que no existen conflictos de intereses.

Recibido: 16 de marzo de 2016 Aceptado: 28 de noviembre de 2016

Correspondencia a: Javier Arturo Hall-López javierhall@uabc.edu.mx 
En relación al rendimiento deportivo, la capacidad aeróbica es un componente crucial de la aptitud física de los atletas y el consumo máximo de oxígeno $\left(\mathrm{VO}_{2}\right.$ máx $)$ un criterio formalmente aceptado para medir la capacidad aeróbica $^{11}$. Teóricamente, algunos autores consideran que la máxima capacidad aeróbica de una persona, es el punto en que el $\mathrm{VO}_{2}$ máx alcanza una meseta a pesar de nuevos incrementos en las cargas de trabajo ${ }^{12}$. Además es considerado como el indicador fisiológico más valido de la función cardiovascular de deportistas determinado en un laboratorio ${ }^{13}$.

En la inmunología aplicada a las ciencias del deporte se refieren diversos modelos que tratan de explicar la relación entre la duración e intensidad del ejercicio y la mayor o menor susceptibilidad a enfermedades del TRS ${ }^{14}$, como los son los denominados modelo de la ventana abierta, modelo $\mathrm{J}$ y modelo neuroendocrino que mencionan que después de 3 a $72 \mathrm{~h}$ de ejercicio, las bacterias y los virus tienen una ventana de oportunidad para entrar con mayor facilidad en el cuerpo 9 .

Recientemente se ha relacionado con dicha teoría al evaluar la respuesta de la inmunoglobulina $\mathrm{A}$ al ejercicio agudo en atletas de alto rendimiento de diversas modalidades deportivas ${ }^{15-18}$. Sin embargo, de acuerdo a nuestro conocimiento, al revisar el estado del arte existen pocas referencias que expliquen la respuesta de la inmunoglobulina A de acuerdo al $\mathrm{VO}_{2}$ máx de una persona, tomando en cuenta la alteración de la homeostasis en el ejercicio agudo $^{7}$ y su recuperación en un ciclo de $24 \mathrm{~h}$. En este contexto, el propósito del presente estudio fue comparar la cinética en la concentración de inmunoglobulina $\mathrm{A}$ salival (IgAs) en adultos jóvenes con capacidad aeróbica promedio o excelente antes y después de una prueba de esfuerzo.

\section{Material y Métodos}

\section{Muestra y selección de participantes}

El presente estudio se realizó bajo un diseño metodológico transversal, con muestreo no probabilístico por conveniencia ${ }^{19}$, siguiendo los principios éticos de investigación en seres humanos de la declaración de Helsinki ${ }^{20}$. Treinta y seis sujetos fueron reclutados a partir de una invitación para participar de manera voluntaria hecha a estudiantes de género masculino de la Facultad de Deportes de la Universidad Autónoma de Baja California. A quienes aceptaran participar, se les programó una cita en el Laboratorio de Biociencias de la Motricidad Humana de dicha institución para solicitarles información general de su historial de salud, determinar el peso $(\mathrm{kg})$ y la estatura $(\mathrm{cm})$ y realizar un electrocardiograma en reposo para descartar algún tipo de condición cardiovascular que les impidiera participar en el estudio. La evaluación del electrocardiograma de 12 derivaciones en reposo se realizó con el equipo General Electric Company, modelo MAC 1200 ST ECG System, Freiburg, Alemania ${ }^{\circledR}$, . Luego fueron evaluados por un médico, descartando algún tipo de condición cardiovascular que les impidiera participar en una prueba de esfuerzo, como lo recomienda el Colegio Americano de Medicina del Deporte (ACSM) ${ }^{21}$.

Para participar en el estudio como criterio de inclusión se consideró aquellos sujetos que tuvieran un valor de $\mathrm{VO}_{2}$ máx entre 38 y 56, que corresponde a las categoría de promedio y excelente. Se determinaron los valores del VO2máx al aplicarles a los sujetos la prueba de campo denominada test course navette de 20 metros $^{22}$ la cual establece la capacidad aeróbica de manera indirecta indicando el $\mathrm{VO}_{2}$ máx. Se les pidió a los participantes vestir short y camiseta para una mayor comodidad en la mecánica de carrera, no realizar ejercicio físico 24 $\mathrm{h}$ antes de la prueba, mantener una buena hidratación y no beber líquidos ni consumir alimentos cuatro horas antes de la evaluación. Para estos efectos, les citó en la pista de atletismo de la Universidad Autónoma de Baja California donde se estableció una marca de inicio y fin de una distancia de 20 metros en el suelo de forma transversal, para que los sujetos se desplazaran corriendo en esa distancia en ida y vuelta bajo la instrucción de un ritmo dado por un sonido emitido de una grabación, con una velocidad de desplazamiento inicial $8.5 \mathrm{~km} \mathrm{~h}-1$ que se incrementó de manera progresiva cada minuto de acuerdo al sonido. La prueba finalizó cuando los sujetos no podían terminar los desplazamientos de carrera con el ritmo y no alcanzando a llegar a la línea y/o su retiro voluntaria de prueba derivado de la fatiga. Una vez terminada la prueba el evaluador registró los niveles y vueltas realizados por los sujeto y de acuerdo a estos valores determinar el valor del $\mathrm{VO}_{2}$ máx y su clasificación.

De los 36 sujetos evaluados sólo 16 participantes cumplieron con el criterio de inclusión; no obstante, sólo nueve accedieron a participar en el estudio: cinco sujetos con capacidad aeróbica promedio y cuatro con capacidad aeróbica excelente, a los cuales se les realizó un prueba de esfuerzo máximo en laboratorio y se evaluó la IgAs mediante la técnica de expectoración pasiva, de manera inicial en reposo, previo a la prueba de esfuerzo y luego, inmediatamente al finalizar la prueba de esfuerzo máximo en cinco momentos posteriores. A continuación en los procedimientos se detallan las evaluaciones.

\section{Procedimientos}

Recolección de saliva mediante la técnica de expectoración pasiva. Para realizar este procedimiento se tomaron en cuenta los criterios establecidos por el Salimetrics Saliva Collection and Handling Advice, traducido del inglés como Manual Guía de Colección de Saliva de Salimetrics $^{23}$.

Se solicitó a los sujetos no consumir alimentos 60 
min antes de la recolección de la muestra y no lavarse o cepillarse los dientes tres horas antes de que la muestra fuese a ser recolectada.

Previo a la evaluación se identificó escribiendo la fecha, código del sujeto y de la evaluación, en un tubo de colección para la muestra falcón y se pesó en la báscula analítica antes de la recolección de la muestra y anotar su peso en la bitácora.

La técnica de expectoración pasiva practicada consiste en lo siguiente: los sujetos deben enjuagarse la boca con agua durante 10 segundos y posteriormente evacuar el contenido. Se pide a los participantes esperar por $10 \mathrm{~min}$ sin hablar, esto para que el agua que utilizan no diluya la saliva; posteriormente se les indica permanecer relajados y sentarse con su cabeza inclinada hacia el frente tratando de recolectar la mayor saliva posible del área sub lingual de la boca, durante dos minutos sin hacer clase alguna de estímulo y realizando el mínimo movimiento oro- facial posible. Al término de los dos minutos se les da en la mano el tubo colector falcón para que lo coloquen en su boca y deslicen la saliva dentro del tubo, se les solicita no tocar el tubo con alguna parte de la boca al depositar la saliva, no respirar en el tubo y no escupir la saliva en el tubo ya que eso puede contaminar las muestra y dar datos erróneos al analizar los datos.

Se recolectaron de cada sujeto 3 a 5 mililitros de saliva y se procedió a cerrar el tubo y pesarse nuevamente para calcular el volumen y registrar el valor final del contenido, determinando el volumen de secreción por minuto. Una vez teniendo la muestra en el tubo falcón se congeló a -20 grados centígrados, para que en una posterior fase de la investigación se determinaran los valores de la IgAs en $\mu \mathrm{g} / \mathrm{mL}$.

\section{Pruebas de esfuerzo máximo}

Los procedimientos de la prueba de esfuerzo consistieron en lo siguiente: El equipo de medición utilizado para realizar las pruebas de esfuerzo máximo incluyó una banda sin fin, ergometro COSMED modelo T-170 $\left(\right.$ COSMED Inc. Italia ${ }^{\circledR}$ ) y para la medición del $\mathrm{VO}_{2}$ máx se utilizó calorimetría indirecta mediante un carro metabólico modelo Quark CPET (COSMED Inc. Italia ${ }^{\circledR}$ ). Este equipo fue calibrado con una mezcla de gases de conocida concentración según las especificaciones del fabricante $\left(\mathrm{CO}_{2}=5 \%, \mathrm{O}_{2}=16 \%\right.$, Balance de $\left.\mathrm{N}_{2}\right)$. La frecuencia cardiaca fue medida con un dispositivo telemétrico marca (COSMED Inc. Italia $\left.{ }^{\circledR}\right)$

Previo a las pruebas de esfuerzo se realizó la primera recolección de saliva mediante la técnica de expectoración pasiva y luego una familiarización del sujeto para utilizar un ergometro de banda sin fin COSMED, modelo T-170 (COSMED Inc. Italia $\left.{ }^{\circledR}\right)$. La prueba consistió en un calentamiento de cinco minutos a una velocidad de $5 \mathrm{~km} /$ $\mathrm{h}$ con $0 \%$ de inclinación; aquí se le mostraba al sujeto el funcionamiento normal del ergometro y se le aconsejó para que corriera lo más cómodo y seguro posible, además se le indicaron procedimientos de seguridad y comunicación con el evaluador.

Los participantes ejecutaron el protocolo de Bruce; que consiste en iniciar con una etapa a velocidad 2,7 $\mathrm{km} / \mathrm{h}$ y una inclinación de $10 \%$; al cabo de $3 \mathrm{~min}$, la velocidad y la pendiente cambian a $4,0 \mathrm{~km} / \mathrm{h}$ a $12 \%, 5,5$ $\mathrm{km} / \mathrm{h}$ a $14 \%, 6,8 \mathrm{~km} / \mathrm{h}$ a $16 \%, 8,0 \mathrm{~km} / \mathrm{h}$ a $18 \%, 8,9 \mathrm{~km} / \mathrm{h}$ a $20 \%, 9,7 \mathrm{~km} / \mathrm{h}$ a $22 \%$, y la última etapa a $10,5 \mathrm{~km} / \mathrm{h}$ a una inclinación de $24 \%$.

Los criterios que se consideraron para finalizar la prueba y determinar que los sujetos alcanzaran el $\mathrm{VO}_{2}$ máx fueron los siguientes: uno subjetivo y dos objetivos:

- Solicitud del sujeto para finalizar la prueba

- Valores de cociente respiratorio entre el $\mathrm{VO}_{2}$ y el $\mathrm{VCO}_{2}$ $(\mathrm{RER}) \geq 1.15 ; \mathrm{y} / \mathrm{o}$

- Valores de $\mathrm{VO}_{2} \leq 2 \mathrm{ml} \cdot \mathrm{kg}-1 \cdot \mathrm{min}-1$ con un cambio de etapa $^{24,25,26}$.

Una vez alcanzado el $\mathrm{VO}_{2}$ máx, se permitió un período de recuperación, donde se observaba al sujeto hasta que alcanzara $80 \%$ de la FCmáx en lat·min-1 predicha para su edad. A partir de ese momento, mediante la técnica de expectoración pasiva, se recolectaron en los sujetos muestras de saliva en cinco ocasiones, inmediatamente finalizada la prueba, $+60 \mathrm{~min},+120 \mathrm{~min},+240 \mathrm{~min} \mathrm{y}$ +1.440 min después la prueba de esfuerzo máximo.

Análisis de la saliva para determinar los valores de la inmunoglobulina A, mediante la técnica de ensayo por inmunoabsorción ligado a enzimas (ELISA). Los análisis químicos se llevaron a cabo en las instalaciones del Laboratorio de Análisis Clínicos del Centro de Diagnóstico de la Facultad de Medicina de la Universidad Autónoma de Baja California. Se procedió a descongelar la saliva colectada por la técnica de expectoración pasiva de cada sujeto en la fase anterior al estudio dejándose a temperatura ambiente del laboratorio; pronto se traspasó del tubo falcón mediante pipetas serológicas o de precisión a un tubo Eppendorf de $2 \mathrm{ml}$ y posteriormente se le agitó. Terminado este proceso se realizaron las alícuotas colocando en cada tubo $250 \mu 1$. Para determinar la variable de IgAs se utilizó el Salimetrics Salivary Secretory IgA Enzyme Immunoassay $\mathrm{Kit}^{\circledR}$ siguiendo las instrucciones del fabricante mediante la técnica de ensayo por inmunoabsorción ligado a enzimas (ELISA).

De acuerdo a la metodología utilizada en la investigación, establecieron hipótesis alterna y nula, teniendo como criterio de aceptación o relación el nivel de $\mathrm{p}<0,05$.

\section{Hipótesis alterna}

El presente estudio anticipa que se encontrarán diferencias en la IgAs en adultos jóvenes con diferente capacidad aeróbica después de una prueba de esfuerzo. 
Tabla 1. Estadística descriptiva de los participantes de acuerdo a la clasificación de la capacidad aeróbica. Los valores representan la Media \pm DE

\begin{tabular}{|c|c|c|c|c|c|}
\hline \multirow[t]{3}{*}{ Variable } & \multicolumn{4}{|c|}{ Capacidad aeróbica } & \multirow[t]{3}{*}{$p=$} \\
\hline & \multicolumn{2}{|c|}{ Promedio (n: 5) } & \multicolumn{2}{|c|}{ Excelente (n: 4) } & \\
\hline & $M \pm D E$ & $\begin{array}{c}\text { Test } \\
\text { Shapiro-Wilk }\end{array}$ & $M \pm D E$ & $\begin{array}{c}\text { Test } \\
\text { Shapiro-Wilk }\end{array}$ & \\
\hline Edad (años) & $21,2 \pm 2,4$ & 0,294 & $21,5 \pm 2,1$ & 0,995 & 0,849 \\
\hline Peso (kg) & $79,2 \pm 8,6$ & 0,742 & $68,5 \pm 3,1$ & 0,24 & 0,048 \\
\hline Estatura $(\mathrm{cm})$ & $174,0 \pm 6,2$ & 0,253 & $174,8 \pm 4,9$ & 0,86 & 0,849 \\
\hline alMC $\left(\mathrm{kg} / \mathrm{m}^{2}\right)$ & $26,1 \pm 3,0$ & 0,776 & $22,4 \pm 1,3$ & 0,72 & 0,058 \\
\hline${ }^{\mathrm{b} F C_{\text {rep }}}$ (lat/min) & $67,4 \pm 5,5$ & 0,576 & $65,0 \pm 5,9$ & 0,43 & 0,555 \\
\hline${ }^{\mathrm{C}} \mathrm{FC} \mathrm{C}_{\max }$ (lat/min) & $190,8 \pm 5,8$ & 0,469 & $183,8 \pm 2,4$ & 0,22 & 0,057 \\
\hline${ }^{d}$ RER & $1,5 \pm 0,2$ & 0,624 & $1,3 \pm 0,0$ & 0,855 & 0,049 \\
\hline eVE $/ \mathrm{NCO}_{2}$ & $26,9 \pm 3,76$ & 0,432 & $30,7 \pm 2,21$ & 0,836 & 0,438 \\
\hline $\mathrm{fVO}_{2}$ máx ml $\cdot \mathrm{kg}^{-1} \cdot \mathrm{min}^{-1}$ & $41,0 \pm 2,7$ & 0,437 & $51,4 \pm 3,2$ & 0,35 & $0,002^{*}$ \\
\hline
\end{tabular}

Nota: Tabla comparativa de la media, desviación estándar ( \pm ) normalidad y homogeneidad de la varianza entre grupos mediante el test estadístico Shapiro-Wilk, * $p<0.05$ : prueba t student para grupos independientes (capacidad aeróbica promedio o excelente). IMC $\left(\mathrm{kg} / \mathrm{m}^{2}\right)$ : ándice de

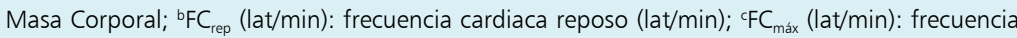
cardiaca máxima (lat/min); dRER: cociente respiratorio entre el $\mathrm{VO}_{2}$ y el $\mathrm{VCO}_{2} ;$ eVENCO2: tasa de ventilación y eliminación de dióxido de carbono; ${ }^{\mathrm{V} V \mathrm{O}_{2}} \mathrm{máx} \mathrm{ml} \cdot \mathrm{kg}^{-1} \cdot \mathrm{min}-1$ : consumo máximo de oxígeno.

\section{Resultados}

En el estudio participaron nueve hombres jóvenes con una edad promedio de $21,3 \pm 2,1$. En la Tabla 1 se muestra la estadística descriptiva de las características físicas y variables fisiológicas antes y después de realizar la prueba de esfuerzo para los grupos, de acuerdo a su capacidad aeróbica, así como la significancia estadística de la prueba $\mathrm{t}$ student para grupos independientes de las variables.

La prueba de ANOVA 2 × 6 mixta indicó que no existieron interacciones significativas entre los grupos y las mediciones $(p=0,956)$. Es decir, no hay un cambio en la concentración de la IgAs a través del tiempo en función de la capacidad aeróbica de los participantes. Tampoco se encuentra una diferencia significativa en la media de la IgAs en los grupos de participantes (promedio $=85,4$ $\pm 19,3$ vs. excelente $=79,2 \pm 21,5 \mu \mathrm{g} / \mathrm{mL}, \mathrm{p}=0,836$ ), independientemente de las mediciones realizadas. Se encuentra una diferencia significativa en la media de las mediciones de IgAs independientemente de la capacidad aeróbica de los participantes $(\mathrm{p}=0,001)$. El análisis post hoc de Tukey indicó que las diferencias se encontraban la medición obtenida inmediatamente después de la prueba y entre la medición inicial $(\mathrm{p}=0,020)$, la medición obtenida $60 \min (\mathrm{p}=0,030), 240 \min (\mathrm{p}=0,016)$ y $1.440 \min (\mathrm{p}=$ $0,028)$ posteriores a la prueba (Figura 1).

\section{Discusión}

De acuerdo a los resultados obtenidos al comparar la cinética en la concentración de (IgAs) en adultos jóvenes con capacidad aeróbica promedio o excelente, antes y después de una prueba de esfuerzo, se observó que no hubo un cambio en la concentración de IgAs a través del tiempo en función de la capacidad aeróbica de los participantes; sin embargo al comparar los valores de IgAs en reposo con los resultados inmediatamente finalizada la prueba de esfuerzo fueron estadísticamente significativos entre los adultos jóvenes con capacidad aeróbica promedio $(147,32 \pm 81,39)$ y los sujetos con capacidad aeróbica excelente $(141,65 \pm 123,16)$. Por otra parte, al comparar las cifras de las variables fisiológicas en los grupos obtenidas de la pruebas de esfuerzo utilizando el protocolo de Bruce, los valores obtenidos en la frecuencia cardiaca máxima (lat $/ \mathrm{min}$ ); cociente respiratorio entre el $\mathrm{VO}_{2}$ y el $\mathrm{VCO}_{2}$ y tasa de ventilación y eliminación de dióxido de carbono, fueron más eficientes físicamente para alcanzar el $\mathrm{VO}_{2}$ máx en el grupo adultos jóvenes con capacidad aeróbica excelente, a pesar que la diferencia en minutos de la prueba de esfuerzo fue mayor (capacidad aeróbica promedio 13,7 $\pm 0,59 \mathrm{~min}$, capacidad aeróbica excelente 15,3 $\pm 0,39 \mathrm{~min}$ ). Esta información es relevante dado que de acuerdo al modelo $\mathrm{J}$ explica que 


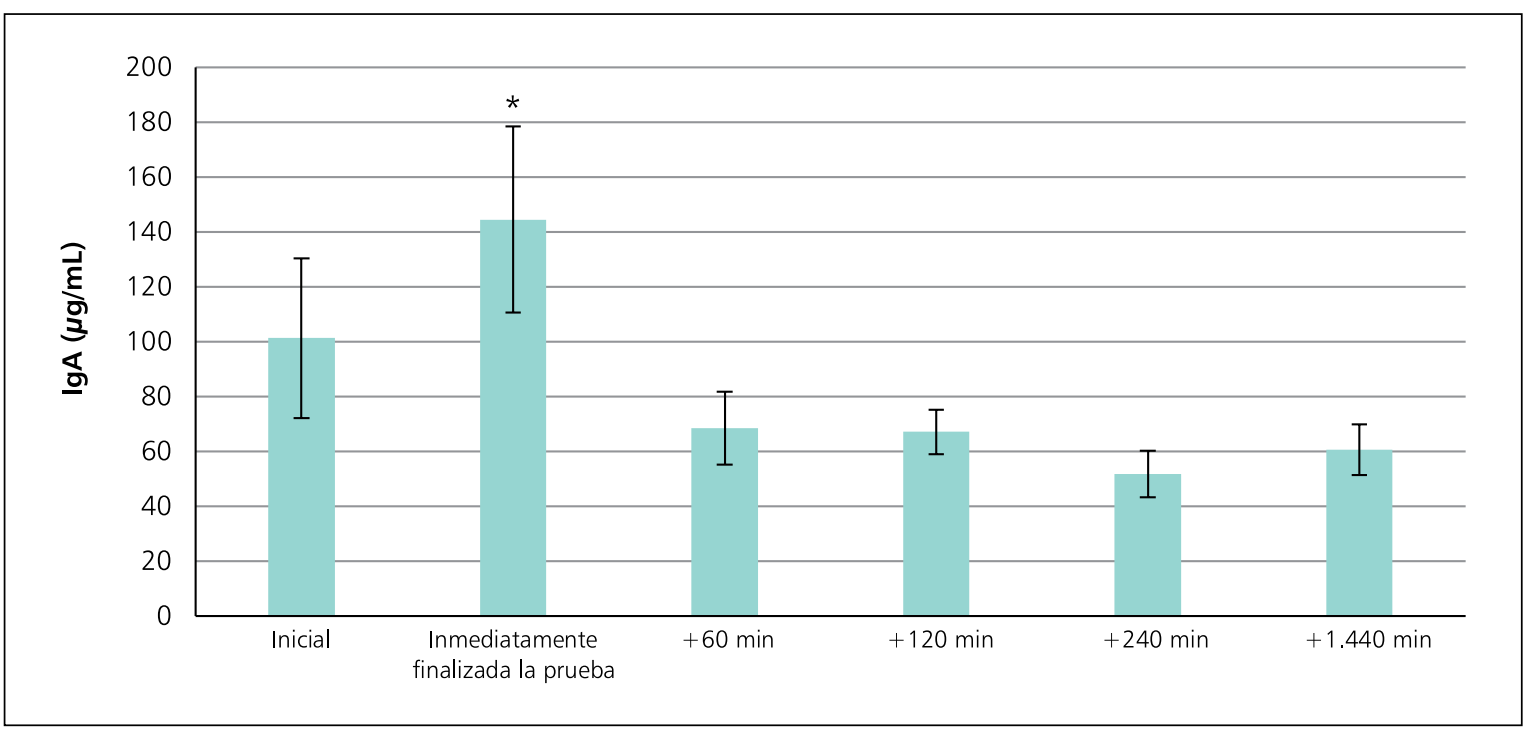

de 3 a 72 h después de realizar ejercicio intenso existe mayor susceptibilidad a enfermedades del TRS, por lo que los resultados encontrados en los sujetos evaluados con capacidad aeróbica promedio en relación a los sujetos con capacidad aeróbica excelente van de acuerdo con el modelo teórico ${ }^{14}$. Por lo cual, en el ámbito de las ciencias aplicadas al deporte, la presente información puede ser útil y prestar atención en las diferencias de las personas en cuanto al nivel de capacidad aerobia al planear un programa de acondicionamiento físico, tendiendo a evitar el síndrome de sobre-entrenamiento en una de sus manifestaciones fisiológicas como lo es la alteración inmunológica ${ }^{1,27,28}$.

Se ha demostrado en estudios transversales comparativos que personas que realizan ejercicio aerobio con intensidad moderada presentan un menor decline de porcentaje de citocinas e $\operatorname{IgA}{ }^{29,30}$. Por otra parte, diversos estudios han evaluado la IgAs en atletas de alto rendimiento de diversas modalidades deportivas aeróbicas y anaeróbicas siguiendo un patrón similar al del presente estudio al realizar evaluación después finalizar un evento deportivo como lo es Jiu-Jitsu, halterofilia y carrera de 5.000 metros planos $^{15-17}$. Otra investigación realizada con un diseño metodológico similar al del presente estudio, pero con un solo grupo de 8 sujetos participantes con edad $21,3 \pm 1,0$ años, masa corporal $66,8 \pm 2,0 \mathrm{~kg}$, y consumo máximo de oxigeno de 52,5 $\pm 2,1 \mathrm{ml} \cdot \mathrm{kg}-1 \cdot \mathrm{min}-1$, evaluó los efectos del ejercicio agudo intenso y prolongado en la modalidad de ciclo ergometro manteniendo un pedaleo a $(143 \pm 4$ watts) por dos horas a $55 \%$ de su capacidad aerobia. La cinética de la IgAs antes, inmediatamente después, 60 min y 180 min después, arrojó resultados similares al no encontrar diferencias significativas ${ }^{18}$.
La presente investigación contiene limitaciones por el tamaño de la muestra y aborda sólo adultos jóvenes físicamente activos de un solo género. También, a pesar que el diseño fue longitudinal y con los resultados no se puede inferir causalidad, no se consideró controlar o medir como co-variables factores de alimentación ${ }^{4}$, los que infieren en el comportamiento de la IgAs, dado que hubiera podido dar elementos de discusión para establecer relación a las adaptaciones fisiológicas agudas derivadas de la prueba de esfuerzo. A pesar de estas limitaciones, se utilizó como equipo de evaluación la calorimetría indirecta considerada como estándar de oro en la evaluación de la capacidad aeróbica.

\section{Conclusión}

Los datos encontrados sugieren que no hay un cambio en la concentración de IgAs a través del tiempo en función de la capacidad aeróbica de los participantes, obteniendo información importante e innovadora que contribuye a una mejor comprensión para los profesionales que trabajan en las ciencias aplicadas al deporte, que contribuye a una mejor comprensión al momento de realizar intervenciones y tomar en cuenta factores de capacidad aeróbica e inmunológicos en los sujetos al momento de prescribir ejercicio físico.

Agradecimientos. A los sujetos participantes por su tiempo y disposición para contribuir con la ciencia. Por el financiamiento en esta investigación al Programa de Intercambio y Cooperación Internacional de Estudios entre Europa y Sudamérica Erasmus Mundus PRECIOSA 2014 y a la Universidad Autónoma de Baja California UABC.
Figura 1. Concentraciones de IgA salival en hombres jóvenes $(n=9)$. 


\section{Resumen}

Introducción. Objetivo: Comparar la cinética en la concentración de inmunoglobulina A salival (IgAs) en adultos jóvenes con capacidad aeróbica promedio (n: 5) o excelente (n: 4) antes y después de una prueba de esfuerzo. Método: 9 adultos jóvenes (edad 21,3 $\pm 2,1)$, divididos de acuerdo su $\mathrm{VO}_{2}$ máx, realizaron una prueba de esfuerzo mediante el protocolo de Bruce. La concentración de IgAs fue determinada mediante el Salimetrics IgA Kit ${ }^{\circledR}$, evaluando inicial, inmediatamente finalizada la prueba, $+60,+120,+240,+1.440$ min. Resultados: La prueba ANOVA 2x6 mixta indicó que no existieron interacciones significativas entre grupos y mediciones $(p=0,956)$. Tampoco se encontró una diferencia significativa en la media de IgAs en los grupos (Promedio $=85,4 \pm 19.3 \mu \mathrm{g} / \mathrm{mL}$ vs Excelente $=79,2 \pm 21.5 \mu \mathrm{g} / \mathrm{mL}, \mathrm{p}=0,836)$. Independientemente de las mediciones, el análisis post hoc de Tukey indicó que las diferencias se encontraron en la medición obtenida inmediatamente después de la prueba y entre la medición inicial (p: 0,020), la medición obtenida $60 \mathrm{~min}$ (p: 0,030), $240 \min (\mathrm{p}: 0,016)$ y $1.440 \min (\mathrm{p}=0,028)$ posteriores a la prueba. Conclusión: Los datos encontrados sugieren que no hay un cambio en la concentración de IgAs a través del tiempo en función de la capacidad aeróbica de los participantes.

\section{Referencias bibliográficas}

1.- Meeusen R, Duclos M, Foster C, Fry A, Gleeson M, Nieman D, et al. Prevention, diagnosis, and treatment of the overtraining syndrome: joint consensus statement of the European College of Sport Science and the American College of Sports Medicine. Med Sci Sports Exerc 2013; 45 (1): 186-205.

2.- Gleeson M. Biochemical and immunological markers of over-training. J Sports Sci Med 2002; 1 (2): 31-41.

3.- Gleeson M, Walsh NP, British Association of $\mathrm{S}$, Exercise $\mathrm{S}$. The BASES expert statement on exercise, immunity, and infection. J Sports Sci 2012; 30 (3): 321-4.

4.- Trochimiak T, Hubner-Wozniak E. Effect of exercise on the level of immunoglobulin $\mathrm{A}$ in saliva. Biol Sport 2012; 29 (4): 255-61

5.- Walsh N P, Gleeson M, Shephard R J, Gleeson M, Woods J A, Bishop N C, et al. Position statement. Part one: Immune function and exercise. Exerc Immunol Rev 2011; 17: 6-63.

6.- Walsh N P, Gleeson M, Pyne D B, Nieman D C, Dhabhar F S, Shephard R J, et al. Position statement. Part two: Maintaining immune health. Exerc Immunol Rev 2011; 17: 64-103.

7.- Wilmore J, Costill D, Kenney L W. Physiology of Sport and Exercise (4th ed.). Champaign, IL: Human Kinetics. 2008.

8.- Nieman DC. Exercise immunology: practical applications. Int J Sports Med 1997; 18 (1): 91-100.

9.- Martin S A, Pence B D, Woods J A. Exercise and respiratory tract viral infections. Exerc Sport Sci Rev 2009; 37 (4): 157-64.

10.- Nieman D C. Exercise, infection, and immunity. Int J Sports Med 1994; 15 (3): 131-41.

11.- Marsh C E. Evaluation of the American College of Sports Medicine submaximal treadmill running test for predicting VO2max. J Strength Cond Res 2012; 26 (2): 548-54.

12.- Astorino T A, White A C. Assessment of anaerobic power to verify $\mathrm{VO} 2 \mathrm{max}$ attainment.
Clin Physiol Funct Imaging 2010; 30 (4): 294-300.

13.- Garber C E, Blissmer B, Deschenes M R, Franklin B A, Lamonte M J, Lee I M, et al. American College of Sports Medicine position stand. Quantity and quality of exercise for developing and maintaining cardiorespiratory, musculoskeletal, and neuromotor fitness in apparently healthy adults: guidance for prescribing exercise. Med Sci Sports Exerc 2011; 43 (7): 1334-59.

14.- Borges G F, Rama L M, Teixeira A M. Models on the relationship between exercise and the risk of upper respiratory tract infections. $\mathrm{R} \mathrm{Ci}$ Med Biol Salvador 2012; 11 (3): 322-5.

15.- Moreira A, Franchini E, de Freitas C G, Schultz de Arruda A F, de Moura N R, Costa E C, et al. Salivary cortisol and immunoglobulin A responses to simulated and official Jiu-Jitsu matches. J Strength Cond Res 2012; 26 (8): 2185-91.

16.- Li C Y, Hsu G S, Suzuki K, Ko M H, Fang S H. Salivary immuno factors, cortisol and testosterone responses in athletes of a competitive 5,000 $\mathrm{m}$ race. Chin J Physiol 2015; 58 (4): 263-9.

17.- Tsai M L, Li T L, Chou L W, Chang C K, Huang S Y, Fang S H. Resting salivary levels of $\operatorname{IgA}$ and cortisol are significantly affected during intensive resistance training periods in elite male weightlifters. J Strength Cond Res 2012; 26 (8): 2202-8.

18.- Li T L, Rush B. The effects of prolonged strenuous exercise on salivary secretion of IgA subclasses in men. Int J Sports Med 2009; 1 (3): 69-74.

19.- Hopkins W G, Marshall S W, Batterham A M, Hanin J. Progressive statistics for studies in sports medicine and exercise science. Med Sci Sports Exerc 2009; 41 (1): 3-13.

20.- Puri K S, Suresh K R, Gogtay N J, Thatte U M. Declaration of Helsinki, 2008: implications for stakeholders in research. J Postgrad Med 2009; 55 (2): 131-4.

21.- American College of Sports Medicine.
ACSM's Guidelines for Exercise Testing and Prescription (9th ed.). Philadelphia, PA: Wolters Kluwer, Lippincott Williams \& Wilkins. 2014.

22.- Ramsbottom R, Brewer J, Williams C. A progressive shuttle run test to estimate maximal oxygen uptake. Br J Sports Med 1988; 22 (4): 141-4.

23.- Salimetrics L L C, SalivaBio L L C. Saliva Collection and Handling Advice. 3rd. 2015. Available at: https://www.salimetrics.com/ assets/documents/Saliva_Collection_Handbook. pdf; accessed on 09.01.2015

24.- Leboeuf S F, Aumer M E, Kraus W E, Johnson J L, Duscha B. Earbud-based sensor for the assessment of energy expenditure, HR, and VO2max. Med Sci Sports Exerc 2014; 46 (5): 1046-52.

25.- Bruce R A. Exercise testing of patients with coronary heart disease. Principles and normal standards for evaluation. Ann Clin Res 1971; 3 (6): 323-32.

26.- Moncada-Jiménez J, Grandjean AJ, Alhassan S, Grandjean PW. Cardiorespiratory responses to continuously-graded and ramp treadmill protocols. Int J Sports Med 2015; 5 (2): 80-6.

27.- Davis J A. Direct determination of aerobic power. Physiological assessment of human fitness. In P. J. Maud \& C. Foster (eds.), Champaign, IL: Human Kinetics. 9-17; 1995.

28.- Varela García D, Castiñeiras Cachaza D, Figueroa Dominguez JA. How does the sport and training variables affect maximum oxygen pulse? Retos 2013; 24:39-45.

29.- Teixeira A M, Martins R A, Martins M, Cunha, M R. Changes in functional fitness, mood states and salivary IgA levels after exercise training for 19 weeks in elderly subjects. Int J Sports Med 2008; 20 (2): 16-26.

30.- Hall-López J, Ochoa-Martínez P, Teixeira A M, Moncada-Jiménez JA, Dantas E. Efecto del ejercicio físico de hidrogimnasia sobre la concentración sérica de inmunoglobulina A en mujeres adultas mayores. Rev Chilena Infectol 2015; 32 (3): 272-7. 